\title{
Molecular targeted therapies for microscopic polyangiitis and granulomatosis with polyangiitis
}

\author{
Masayoshi Harigai ${ }^{1}$, Michi Tsutsumino $^{2}$, Hideto Takada ${ }^{2}$, and Kenji Nagasaka ${ }^{3}$
}

${ }^{1}$ Division of Epidemiology and Pharmacoepidemiology, ${ }^{2}$ Department of Rheumatology, School of Medicine, Tokyo Women's Medical University, Tokyo; ${ }^{3}$ Department of Rheumatology, Ome Municipal General Hospital, Tokyo, Japan

Received: October 13, 2018 Accepted: November 17, 2018

\section{Correspondence to}

Masayoshi Harigai, M.D.

Division of Epidemiology and Pharmacoepidemiology, Department of Rheumatology, School of Medicine, Tokyo Women's Medical University, 10-22 Kawada-cho, Shinjuku-ku, Tokyo 162-0054, Japan

Tel: +81-3-5269-1725

Fax: +81-3-5269-9154

E-mail: harigai.masayoshi@

twmu.ac.jp
Clinical trials and observational studies have established cyclophosphamide (CY) or rituximab plus glucocorticoid (GC) as standard remission induction therapies in patients with microscopic polyangiitis (MPA) and granulomatosis with polyangiitis (GPA). However, because these regimens are associated with serious adverse drug reactions, the development of drugs with novel mechanisms of actions are needed. Progress in basic and clinical research has identified novel candidate targeting molecules, including B-cell activating factor (BAF), C5a receptor, and interleukin-6. The combination of rituximab and BAF blockade in patients with MPA and GPA is under investigation in an effort to strike a better benefit-risk balance. Phase II clinical trials of avacopan (CCX168), an orally administered $\mathrm{C}_{5}$ a receptor antagonist, have suggested a reduction in the dosage of concomitant GC or the replacement of GC in patients with MPA and GPA. The results from a currently ongoing phase III trial are awaited. Anecdotal case reports and an open-label pilot study have indicated the effectiveness of tocilizumab in patients with MPA and GPA. A randomized clinical trial comparing tocilizumab and intravenous CY in combination with GC is currently in progress. Molecular targeted therapy is expected to transform the treatment strategy for MPA and GPA to allow GC-free or at least less GC-dependent forms of therapy.

Keywords: Microscopic polyangiitis; Granulomatosis with polyangiitis; Molecular targeted therapy; Interleukin-6; Complement C5a

\section{INTRODUCTION}

Microscopic polyangiitis (MPA) and granulomatosis with polyangiitis (GPA) are systemic small-vessel vasculitides that are part of the group of diseases referred to as anti-neutrophil cytoplasmic antibody (ANCA)-associated vasculitis (AAV). Until the introduction of cyclophosphamide (CY) combined with glucocorticoid (GC), the prognosis of AAV, and particularly of GPA, was very poor $[1,2]$. Recent guidelines for MPA and GPA from the Japan Research Committee of the Ministry of Health, Labour, and Welfare for Intractable Vasculitis (JPVAS), as well as those from the British Society of Rheumatol- ogy (BSR)/British Health Professionals in Rheumatology (BHPR), the European League Against Rheumatism (EULAR)/European Renal Association-European Dialysis and Transplant Association (ERA-EDTA), and the Kidney Disease Improving Global Outcomes (KDIGO) all recommend the use of $\mathrm{CY}$ or rituximab plus $\mathrm{GC}$ as first-line therapy [3-6]. These recommendations are based on initial cohort studies of CY combined with GC $[1,2]$ and the results of several clinical trials in which $\mathrm{CY}$ or rituximab combined with GC was tested.

Despite the established efficacies of CY plus GC and rituximab plus GC for the treatment of MPA and GPA, a substantial proportion of patients fail to show 
an appropriate therapeutic response, as some patients experience disease relapse, even those under remission-maintenance treatment with GC plus azathioprine or other immunosuppressive agents. The use of CY or rituximab plus GC is associated with several serious adverse drug reactions that decrease the quality of life of patients. A possible solution to this clinical challenge is the development of molecular targeted drugs with novel mechanisms of action, a strategy that has markedly improved the outcomes of patients with rheumatoid arthritis [7]. Recent advances in basic and clinical research have accumulated evidence of the efficacy of targeting molecules in patients with MPA and GPA. In this article, we review the efficacy and safety of the standard regimens for MPA and GPA and discuss the molecular targeted drugs under clinical development. Demonstration of similar or improved efficacy of these drugs and their greater safety may enable a decrease in the concomitant dose or even the exclusion of GC for the treatment of MPA and GPA.

\section{CYCLOPHOSPHAMIDE FOR PATIENTS WITH MPA AND GPA}

In a systematic review recently published by JPVAS [8], a randomized controlled trial (RCT) and two cohort studies were evaluated using the Grading of Recommendations Assessment, Development, and Evaluation (GRADE) system. Neither the RCT [9] nor one of the cohort studies [10] revealed a significant difference between GC monotherapy and GC plus CY regarding death, end-stage renal disease, regarding death, endstage renal disease, and remission of MPA or GPA. However, in both studies, the certainty of evidence of each evaluated outcome was downgraded to very low because of the risk of bias and imprecision. In the other cohort study [2], the relative risk (95\% confidence interval [CI]) of GC plus CY for remission versus GC monotherapy was 1.51 (95\% CI, 1.05 to 2.17 ), which was statistically significant. However, the risk of bias and imprecision again resulted in a very low certainty of evidence of the outcome. Despite the lack of sufficient evidence for the superior efficacy of CY plus GC versus GC monotherapy, a spate of clinical trials has demonstrated good clinical outcomes in patients with MPA and GPA treated with CY plus GC [11-15].

Safety concerns regarding the use of CY plus GC pose a challenge to physicians treating patients with MPA and GPA. GC use is associated with serious infection, osteoporosis, osteonecrosis, glaucoma, cardiovascular diseases, cerebrovascular diseases, and glucose intolerance, and $\mathrm{CY}$ is associated with increased risk of serious infection, cytopenia, malignancy, liver dysfunction, and premature gonadal failure. In an observational study of 535 patients who participated in four RCTs conducted by the European Vasculitis Study Group (EUVAS), adverse events including osteoporosis, malignancy, and diabetes were reported at rates exceeding $10 \%$ each. Lower glomerular filtration rates, four or more flares, and longer use of GC were independent risk factors for high-level damage (i.e., Vascular Damage Index z 5) [16]. Consequently, novel agents that produce a better benefit-risk balance than CY plus GC in patients with MPA and GPA are urgently needed.

\section{RITUXIMAB FOR PATIENTS WITH MPA AND GPA}

The anti-human $\mathrm{CD} 20$ chimeric antibody rituximab has been approved in many countries for use in treating patients with MPA and GPA, and in clinical practice guidelines it is recommended as a first-line agent [3-6]. Two RCTs have established the efficacy and safety of rituximab in patients with MPA and GPA (Table 1). In the Rituximab in ANCA-associated Vasculitis (RAVE) trial [15], patients with newly diagnosed or relapsing MPA or GPA were randomly assigned to the rituximab $\left(375 \mathrm{mg} / \mathrm{m}^{2}\right.$ of body surface area, once weekly for 4 weeks) or the control group (daily oral dose of $2 \mathrm{mg} \mathrm{CY} / \mathrm{kg}$ followed by a daily azathioprine dose of $2 \mathrm{mg} / \mathrm{kg}$ ). At month 6 of treatment, rituximab was not inferior to daily oral $\mathrm{CY}$ for the primary endpoint of remission without the use of prednisone ( $64 \%$ vs. $53 \%$, respectively; $p<0.001$ ). In addition, rituximab was more efficacious than $\mathrm{CY}$ among patients with relapsing disease in achieving the primary endpoint ( $67 \%$ vs. $42 \%$, respectively; $p=0.01$ ). The Rituximab versus CY in ANCA-associated Renal Vasculitis (RITUXVAS) trial enrolled patients with newly diagnosed MPA or GPA with renal involvement [14] and then randomized them to the rituximab group, 
Table 1. Major clinical trials of rituximab in patients with MPA and GPA

\begin{tabular}{|c|c|c|c|c|c|}
\hline Trial & Patients & Investigational group & $\begin{array}{l}\text { Control } \\
\text { group }\end{array}$ & $\begin{array}{l}\text { Primary } \\
\text { endpoint }\end{array}$ & Main results ${ }^{\mathrm{a}}$ \\
\hline \multicolumn{6}{|l|}{ Induction trials } \\
\hline RAVE & $\begin{array}{l}\text { Newly diagnosed or } \\
\text { relapsing GPA or MPA }\end{array}$ & $\begin{array}{l}\text { RTX } 375 \mathrm{mg} / \mathrm{m}^{2} \text { once } \\
\text { weekly for } 4 \text { weeks + } \\
\text { GC }(\mathrm{n}=99)\end{array}$ & $\begin{array}{l}\text { Oral CY } 2 \mathrm{mg} / \\
\mathrm{kg} \text { per day }+ \\
\mathrm{GC}(\mathrm{n}=98)\end{array}$ & $\begin{array}{l}\text { Remission } \\
\text { without GC at } \\
\text { month } 6\end{array}$ & $\begin{array}{l}\text { RTX non-inferior } \\
(64 \%) \text { vs. oral CY }(53 \%) \\
\text { RTX superior }(67 \%) \text { vs. } \\
\text { oral CY }(42 \%) \text { in relapsing } \\
\text { patients }\end{array}$ \\
\hline RITUXVAS & $\begin{array}{l}\text { Newly diagnosed GPA } \\
\text { or MPA with renal } \\
\text { involvement }\end{array}$ & $\begin{array}{l}\text { RTX } 375 \mathrm{mg} / \mathrm{m}^{2} \text { once } \\
\text { weekly for } 4 \text { weeks + } \\
\mathrm{GC}+2 \text { doses of } \\
\text { IVCY } 15 \mathrm{mg} / \mathrm{kg}(\mathrm{n}=33)\end{array}$ & $\begin{array}{l}\text { IVCY for } \\
3-6 \text { months } \\
\text { followed by } \\
\text { AZA }(n=11)\end{array}$ & $\begin{array}{l}\text { Sustained } \\
\text { remission at } \\
\text { month } 12 \text { and } \\
\text { severe adverse } \\
\text { events }\end{array}$ & $\begin{array}{l}\text { RTX non-inferior (76\%) } \\
\text { vs. IVCY (82\%) } \\
\text { No difference in severe } \\
\text { adverse events (RTX } 42 \% \\
\text { vs. IVCY } 36 \% \text { ) }\end{array}$ \\
\hline \multicolumn{6}{|l|}{$\begin{array}{l}\text { Maintenance } \\
\text { trials }\end{array}$} \\
\hline MAINRITSAN & $\begin{array}{l}\text { Newly diagnosed or } \\
\text { relapsing GPA or MPA } \\
\text { in complete remission } \\
\text { achieved with IVCY + } \\
\text { GC }\end{array}$ & $\begin{array}{l}\text { RTX } 500 \text { mg on days o } \\
\text { and } 14 \text { and at } \\
\text { months } 6,12 \text {, and } 18 \\
(n=57)\end{array}$ & $\begin{array}{l}\text { AZA } 2 \mathrm{mg} / \mathrm{kg} \\
\text { tapering to } \\
\text { zero at } 22 \\
\text { months } \\
(\mathrm{n}=58)\end{array}$ & $\begin{array}{l}\text { Major relapse } \\
\text { at month } 28\end{array}$ & $\begin{array}{l}\text { RTX superior (5\%) vs. } \\
\operatorname{AZA~(29\% )~}\end{array}$ \\
\hline $\begin{array}{l}\text { MAINRIT- } \\
\text { SAN2 }\end{array}$ & $\begin{array}{l}\text { Newly diagnosed or } \\
\text { relapsing GPA or MPA } \\
\text { in complete remission } \\
\text { achieved with CY or } \\
\text { RTX + GC }\end{array}$ & $\begin{array}{l}\text { RTX } 500 \text { mg on day o } \\
\text { and reinfusion based } \\
\text { on ANCA and CD19+ } \\
\text { lymphocytes until } \\
\text { month } 18 \\
\text { (tailored-infusion, } \\
\mathrm{n}=81 \text { ) }\end{array}$ & $\begin{array}{l}\text { RTX } 500 \text { mg } \\
\text { on days } \\
\text { o and } 14 \\
\text { and at } \\
\text { months } 6 \text {, } \\
\text { 12, and } 18 \\
\text { (fixed } \\
\text { schedule, } \\
\mathrm{n}=81 \text { ) }\end{array}$ & $\begin{array}{l}\text { Relapse at } \\
\text { month } 28\end{array}$ & $\begin{array}{l}\text { No difference in relapse } \\
\text { rates (tailored-infusion } \\
\text { 17.3\% vs. fixed infusion } \\
9.9 \% \text { ) } \\
\text { Fewer rituximab } \\
\text { infusions in tailored- } \\
\text { infusion patients than } \\
\text { fixed-schedule patients } \\
\text { (medians of } 3 \text { vs. } 5 \text {, } \\
\text { respectively) }\end{array}$ \\
\hline
\end{tabular}

MPA, microscopic polyangiitis; GPA, granulomatosis with polyangiitis; RAVE, Rituximab in ANCA-associated Vasculitis; RTX, rituximab; GC, glucocorticoid; CY, cyclophosphamide; RITUXVAS, Rituximab versus CY in ANCA-associated Renal Vasculitis; IVCY, intravenous cyclophosphamide; AZA, azathioprine; MAINRITSAN, Maintenance of Remission using Rituximab in Systemic ANCA-Associated Vasculitis; ANCA, anti-neutrophil cytoplasmic antibody.

${ }^{a}$ RAVE and RITUXIVAS are designed as non-inferiority trials.

which received rituximab with two doses of intravenous (IV) CY, or the control group, which received only IVCY for 3 to 6 months followed by treatment with azathioprine. Sustained-remission rates at month 12 were similar between the rituximab and control groups $(76 \%$ vs. $82 \%$, respectively; $p=0.68)$. In both the RAVE and the RITUXVAS trials, the two groups of patients did not significantly differ in the number of severe adverse events. The long-term follow-up of patients enrolled in these trials showed that the relapse rates in patients treated with a single course of rituximab without maintenance therapy were similar to those in patients treat- ed with CY followed by azathioprine maintenance therapy: $32 \%$ versus $29 \%$, respectively, at month 18 in the RAVE trial and $21 \%$ versus $18 \%$, respectively, at month 24 in the RITUXVAS trial $[17,18]$.

The Maintenance of Remission using Rituximab in Systemic ANCA-Associated Vasculitis (MAINRITSAN) trial evaluated the efficacy and safety of rituximab vs. azathioprine for remission maintenance in patients with MPA and GPA (Table 1) [19]. Patients with newly diagnosed or relapsing GPA, MPA, or renal-limited AAV who had achieved remission with IVCY plus GC were enrolled and then randomly assigned to re- 
ceive either $500 \mathrm{mg}$ rituximab on days $\mathrm{o}$ and 14 and at months 6,12 , and 18 or daily azathioprine at $2 \mathrm{mg} / \mathrm{kg}$ until month 22. Fewer patients had a major relapse at month 28 in the rituximab group than in the azathioprine group ( $5 \%$ vs. $29 \%$, respectively; $p=0.002$ ). A longterm analysis of this trial demonstrated that rituximab remained superior to azathioprine for sustaining remission at month 60 (57.9\% vs. $37.2 \%$, respectively; $p=0.012)[20]$. The subsequent trial, MAINRITSAN2, compared tailored versus fixed-schedule rituximab reinfusion for remission maintenance in patients with MPA and GPA (Table 1) [21]. Patients in the tailored-infusion group received a $500 \mathrm{mg}$ rituximab infusion at randomization, with additional rituximab infusions until month 18 based on ANCA and CD19+ lymphocyte counts. Fixed-schedule patients received $500 \mathrm{mg}$ rituximab infusions on days 0 and 14 and then at months 6 , 12, and 18. The two groups did not significantly differ in terms of the proportion of patients with relapse at month $28: 17.3 \%$ in the tailored-infusion group vs. $9.9 \%$ in the fixed-schedule patients $(p=0.22)$. Patients in the former group received fewer rituximab infusions.

The results from these clinical trials established the similar efficacy and safety of rituximab plus GC compared to CY plus GC in patients with MPA and GPA. Their findings also strongly suggest the involvement of $\mathrm{CD} 2 \mathrm{O}+\mathrm{B}$ cells in the immunopathogenesis of these vasculitides.

\section{B-CELL ACTIVATING FACTOR-TARGETED THERAPY}

Accumulating evidence suggests problems in B-cell depletion therapy when rituximab is used for patients with MPA and GPA, such as a requirement for concomitant high-dose GC in the induction of remission and long-term low-dose GC to prevent relapses. Several studies have reported increases in the levels of B-cell activating factor (BAF) in patients with MPA and GPA [22-24]. Blockade of BAF in combination with rituximab may deplete B-cells more broadly and to a greater extent and may inhibit the recovery of autoreactive B-cells, thereby contributing to the maintenance of remission. A clinical trial to evaluate the efficacy and safety of this combined anti-B-cell therapy approach for AAV is under preparation [25].

\section{COMPLEMENT TARGETED THERAPY}

The approximately 30 molecules that make up the complement system play essential roles in both innate and adaptive immunity [26]. There are three pathways that lead to complement activation: the classic, alternative, and lectin pathways. All three include the formation of $\mathrm{C}_{3} \mathrm{a}, \mathrm{C}_{3} \mathrm{~b}, \mathrm{C} 5 \mathrm{a}$, and the terminal complement complex $\mathrm{C}_{5} \mathrm{~b}_{6789}$. C5a is a potent pro-inflammatory mediator that, after binding to the $\mathrm{C}_{5}$ a receptor (C5aR, CD88), stimulates leukocyte migration, activation, degranulation, vascular permeability, and the release of proteinases and oxidative free radicals. CD88 is expressed by neutrophils, mast cells, basophils, eosinophils, monocytes, and vascular endothelial cells, among others. Although complement deposition is not observed in AAV-related pauci-immune glomerulonephritis, growing evidence strongly suggests the involvement of the complement system in the pathogenesis of AAV [27].

Avacopan is an orally administered antagonist of C5aR that is under clinical development as a treatment for AAV [28-30]. Xiao et al. [30] established a mouse model of ANCA-associated renal vasculitis, in which mouse anti-myeloperoxidase (MPO) immunoglobulin $\mathrm{G}$ (IgG)-induced necrotizing and crescentic glomerulonephritis (NCGN) develops in mice with knockedin human $\mathrm{C}_{5} \mathrm{aR} / \mathrm{CD} 88$ and knocked-out mouse C5aR/ CD88. Oral administration of avacopan almost completely ameliorates anti-MPO IgG-induced NCGN in this model. Along with this dose-dependent response are significant improvements in hematuria, proteinuria, and leukocyturia. In the human myeloid cell line U937, avacopan significantly inhibits the binding of [25I]-C5a to C5aR as well as C5a-induced migration and the intracellular release of calcium [28]. Avacopan also strongly suppresses the binding of [ $\left.{ }^{125} \mathrm{I}\right]-\mathrm{C}_{5}$ a to $\mathrm{C}_{5} \mathrm{aR}$, C5a-induced intracellular calcium release, chemotaxis, the upregulation of CD11b, and the oxidative burst in human neutrophils in vitro. Oral administration of avacopan 1 hour prior to blood sampling significantly inhibits $\mathrm{C}_{5}$ a-induced upregulation of CD11b in neutrophils of mice with knocked-in human C5aR/CD88 [30].

In a phase I study of avacopan that enrolled 48 healthy volunteers to evaluate the safety and tolerability of the drug in humans, avacopan was well tolerated and dose-limiting or serious adverse events were 
not reported [28]. Commonly observed adverse events included diarrhea, dizziness, lower abdominal pain, nausea, and oropharyngeal pain. The average maximal plasma level of avacopan after the administration of 30 mg twice a day was $191 \mathrm{ng} / \mathrm{mL}$, and the terminal halflife was 129 hours. A single $30 \mathrm{mg}$ dose of avacopan or a 7-day regimen of $30 \mathrm{mg}$ twice a day dose-dependently inhibited $\mathrm{C}_{5} \mathrm{a}$-induced $\mathrm{CD} 11 \mathrm{~b}$ expression in peripheral blood neutrophils [28].

In the clinical trial, a randomized, double-blind, placebo-controlled, phase 2 study to evaluate the safety and efficacy of CCX168 in subjects With anti-neutrophil cytoplasmic antibody (ANCA)-associated vasculitis on background of cyclophosphamide or rituximab treatment (CLEAR), patients with newly diagnosed or relapsed AAV received placebo plus prednisone starting at $60 \mathrm{mg}$ daily (control group, $\mathrm{n}=23$ ), $30 \mathrm{mg}$ avacopan twice a day plus reduced prednisone starting at $20 \mathrm{mg}$ daily $(\mathrm{n}=22)$, or $30 \mathrm{mg}$ avacopan twice a day without prednisone $(n=22)$ [29]. All patients received IVCY at a dose of $15 \mathrm{mg} / \mathrm{kg}$ at weeks $\mathrm{O}, 2,4,8$, and 12 followed by azathioprine at a target dose of $2 \mathrm{mg} / \mathrm{kg}$ per day up to week 24 or IV rituximab at $375 \mathrm{mg} / \mathrm{m}^{2}$ per week for 4 weeks. The aim of the study was to determine the feasibility of reducing or replacing GC with avacopan without compromising efficacy in treating AAV. The primary endpoint was the proportion of patients with a decrease in their Birmingham Vasculitis Activity Scores $($ BVAS) $\geq 50 \%$ from baseline and no disease worsening in any body system. Secondary endpoints were the proportion of patients with a renal response, BVAS-confirmed remission, and changes in the BVAS and main laboratory parameters compared to the baseline. Patients in the three groups ranged in age from 57 to 59.1 years old. Of the 67 patients enrolled, 33 (49\%) each had GPA and MPA and one had unknown disease type; 35 (52\%) and $29(43 \%)$ patients were positive for MPO-ANCA and proteinase 3 ( $\left.\mathrm{PR}_{3}\right)$-ANCA, respectively. Almost all patients had renal involvement (97\%) and the mean BVAS of the three groups ranged from 13.2 to 14.3. The primary endpoint was met in 14 of 20 patients (70.0\%) in the control group, 19 of 22 (86.4\%) in the avacopan plus reduced-dose prednisone group, and 17 of $21(81.0 \%)$ in the avacopan without prednisone group. The differences compared to the control group were $16.4 \%$ for the avacopan plus reduced prednisone group (90\% CI, -4.3 to $37.1 ; p=0.002$ ) and $11.0 \%$ for the avacopan without prednisone group (90\% CI, -11.0 to 32.9; $p=0.01$ ), thus demonstrating the non-inferiority of these two groups compared to the control group. Adverse events were reported in 21 of the 23 patients (91\%) in the control group, 19 of the 22 patients (86\%) in the avacopan plus reduced prednisone group, and 21 of the 22 patients (96\%) in the avacopan without prednisone group. Changes in the BVAS tended to be larger in the avacopan-treated groups than in the control group but the difference was not statistically significant, while the changes in the EuroQoL-5D-5L visual analog scale at week 12 were significantly better in the two avacopan groups than in the control group. Short form-36 physical functioning scores, role emotional scores, urinary albumin/creatinine ratios, and urinary monocyte chemoattractant protein-1/creatinine ratios improved significantly at week 12 in the avacopan plus reduced-dose prednisone group versus the control group [29].

Based on the promising results from the phase II study, a multi-national phase III study (ADVOCATE) (ClinicalTrials.gov Identifier: NCTo2994927) is currently in progress. Eligible patients are those with incident or relapsed MPA or GPA with ANCA positivity for which treatment with CY or rituximab is needed; with at least one major item, three non-major items, or two renal items of proteinuria and hematuria according to BVAS; and with an estimated glomerular filtration rate $>15 \mathrm{~mL} / \mathrm{min} / 1.73 \mathrm{~m}^{2}$ at the time of screening. Patients are allocated to either the avacopan plus rituximab or CY (IV or oral) group or to the prednisone plus rituximab or CY group. Following initial treatment, both groups receive azathioprine. The primary endpoints are the proportion of patients achieving disease remission as determined by BVAS at week 26 and the proportion of patients achieving sustained remission assessed by BVAS at week 52 [31]. The demonstrated success of avacopan may open the door to a new era of GC-free AAV treatment.

\section{INTERLEUKIN-6 TARGETED THERAPY}

\section{Interleukin-6 in an AAV animal model and in patients with MPA and GPA}

Interleukin-6 (IL-6) is a pleotropic inflammatory cyto- 
kine that is also involved in the pathogenesis of AAV. Nagao et al. [32] found a significant association between the serum concentration of IL- 6 and glomerular crescent formation in SCG/Kj mice, an animal model of MPA. Treatment of the mice with anti-IL-6-receptor antibody significantly decreased serum titers of MPO-ANCA and tended to improve proteinuria and hematuria. Kekow et al. [33] reported higher serum levels of IL-6 in patients with active MPA and eosinophilic GPA than in normal controls. The production of IL-6 by vascular endothelial cells in vitro is induced by the stimulation with sera positive for ANCA or anti-endothelial cell antibody from patients with AAV [34]. Peripheral blood mononuclear cells stimulated with $\mathrm{PR}_{3}$ or MPO exhibit enhanced production of IL-6 in vitro [35]. In 2004, Ohlsson et al. [36] reported an association between serum levels of cytokines and disease activity in patients with MPA and GPA. They found a significantly higher plasma concentration of IL-6 in AAV patients than in normal controls, irrespective of disease activity, and that patients with active disease have higher IL- 6 concentrations than those with inactive disease. Higher IL-6 levels were also measured in patients with stable disease who experienced disease relapse within 3 months. Tsutsumino et al. [37] measured the serum concentrations of a panel of cytokines and chemokines in patients with MPA and GPA before and after remission induction therapy. Serum concentrations of IL- 6 were elevated in patients with active disease but they significantly and dramatically decreased within 6 months after treatment initiation. Berti et al. [38] reported higher serum levels of IL- 6 in 10 patients with newly developed and active AAV than in normal controls and enhanced expression of IL-6 in both vascular endothelial cells and cells infiltrating affected organs.

\section{Case reports of AAV successfully treated with an- ti-IL-6 receptor antibody}

Anecdotal evidence suggests that IL-6 blockade is therapeutically effective for patients with AAV. Table 2 summarizes the clinical characteristics of patients with AAV treated with tocilizumab as described in the literature, except for patients enrolled in an open-label pilot study, which is described below. Overall, four cases of primary AAV and two cases of AAV and rheumatoid arthritis have been reported.
Takenaka et al. [39] reported the first case of primary AAV with MPO-ANCA in a patient who was resistant to $\mathrm{CY}$ and achieved clinical remission with tocilizumab. A 47-year-old woman with unclassifiable AAV relapsed with aortitis and hypertrophic pachymeningitis, which did not respond to treatment with prednisolone (PSL) and CY. However, after CY was replaced with IV tocilizumab at $8 \mathrm{mg} / \mathrm{kg}$ for 4 weeks, the aortitis improved and the dose of PSL maintaining remission was reduced to $4 \mathrm{mg} /$ day.

Berti et al. [38] reported the case of a 32-year-old man with MPA who developed alveolar hemorrhage and proliferative glomerulonephritis resistant to treatment with GC plus IVCY, methotrexate (MTX), or mycophenolate mofetil. The patient was allergic to rituximab. Treatment with tocilizumab at $8 \mathrm{mg} / \mathrm{kg}$ for 4 weeks resulted in improved signs, symptoms, and laboratory findings. The PSL dose was reduced to $7.5 \mathrm{mg} /$ day. Sakai et al. [40] reported two cases of new-onset AAV treated with tocilizumab. In the first case, a 73-year-old woman developed MPA with active crescent-forming glomerulonephritis and interstitial pneumonia. She received $8 \mathrm{mg} / \mathrm{kg}$ IV tocilizumab every month along with PSL at $50 \mathrm{mg} /$ day $(1 \mathrm{mg} / \mathrm{kg} /$ day), which soon ameliorated her signs, symptoms, and laboratory findings. The PSL dose was decreased to $5 \mathrm{mg}$ /day at month 6 . In the second case, a 76-year-old man with MPA characterized by rapidly progressive glomerulonephritis and pulmonary fibrosis was treated with an IV infusion of $8 \mathrm{mg}$ tocilizumab/kg and started on PSL treatment at $70 \mathrm{mg} /$ day ( 1 $\mathrm{mg} / \mathrm{kg} /$ day). His general symptoms soon improved but he experienced cellulitis on the left calcaneus 3 weeks after the first infusion of tocilizumab, which forced a discontinuation of the drug.

Sumida et al. [41] reported a case of AAV in a 74-yearold woman who developed MPO-ANCA-associated glomerulonephritis while being treated for rheumatoid arthritis with $5 \mathrm{mg}$ PSL/day and $4 \mathrm{mg}$ MTX/week. The administration of $30 \mathrm{mg}$ PSL/day and IVCY improved her condition but the glomerulonephritis relapsed and was resistant to tacrolimus. IV administration of $8 \mathrm{mg}$ tocilizumab/kg per month dramatically improved her signs and symptoms as well as her laboratory findings and allowed PSL tapering.

Sakai et al. [42] also reported a case involving AAV that developed in a 69-year-old man being treated with 


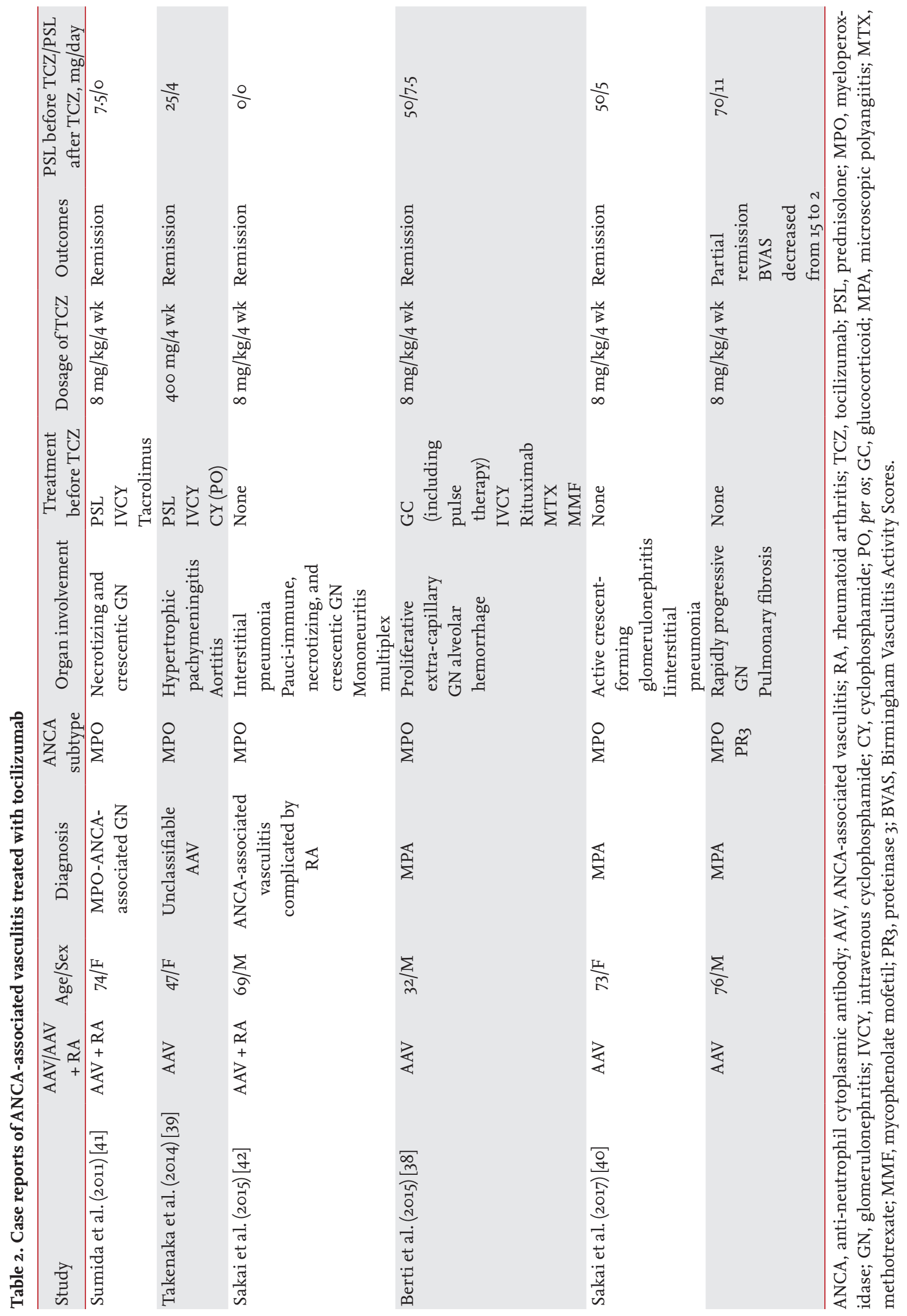


bucillamine for rheumatoid arthritis. The AAV was accompanied by interstitial pneumonia, pauci-immune and crescent-forming glomerulonephritis, and mononeuritis multiplex. Tocilizumab monotherapy at $8 \mathrm{mg} / \mathrm{kg}$ per month markedly improved his general symptoms and laboratory findings, and remission was maintained 1 year after initiation of tocilizumab treatment.

\section{An open-label pilot study of tocilizumab for MPA}

Sakai et al. [42] conducted an open-label pilot study of tocilizumab for MPA. Patients with newly developed MPA classified according to the algorithm of the European Medical Agency [43] were eligible for the study. Patients were treated with tocilizumab monotherapy without GC or immunosuppressant therapy. Tocilizumab was given intravenously at $8 \mathrm{mg} / \mathrm{kg}$ at weeks $\mathrm{o}, 2$, 4,6 , and 8 and every 4 weeks from week 12 onward. Tocilizumab was discontinued after week 48 and the patients, without any further treatment, were observed for MPA for 1 year. All seven patients (five males and two females; mean age of 69.9 years old) had renal involvement, defined as the presence of biopsy-proven vasculitis or active urine sediments. The BVAS and main laboratory data (mean $\pm \mathrm{SD}$ ) were as follows: BVAS, $15.6 \pm$ 7.0; MPO-ANCA, $159.5 \pm 102.7 \mathrm{U} / \mathrm{mL}$; C-reactive protein (CRP), $6.7 \pm 5.3 \mathrm{mg} / \mathrm{dL}$; and serum creatinine, $1.15 \pm 0.49 \mathrm{mg} / \mathrm{dL}$. The primary outcomes consisted of the proportions of patients with complete remission (CR) and partial remission (PR) and the improvement of major clinical signs and laboratory data at month 6 . CR was defined as a BVAS of 0 at two consecutive visits made at least 1 month apart, and PR as a 50\% reduction in the BVAS from baseline. One patient voluntarily discontinued the study at month 3. CR was achieved in two (33.3\%) and PR in four (66.7\%) of the remaining six patients at month 6. MPO-ANCA improved in five of the six remaining patients at month 6 and CRP decreased in all seven patients at month 1 . CY was maintained in three of the six patients after tocilizumab was stopped at month 12; four patients were able to remain drugfree after the 12 months of tocilizumab treatment. Two patients developed respiratory infections during treatment with tocilizumab. Overall, these data show the efficacy of tocilizumab in patients with MPA; however, the relatively low $\mathrm{CR}$ rates suggest the need for adjunctive GC therapy.

\section{Randomized controlled trial of tocilizumab for MPA and GPA}

Based on the available evidence discussed so far, we have launched a multi-center, open, parallel-group RCT of tocilizumab for patients with MPA and GPA, with the aim of achieving drug approval in Japan (Fig. 1). The study is registered with the Clinical Trial Registry of Japan Medical Association with identifier JMAIIAo0325. Its objective is to investigate the efficacy, safety, and pharmacokinetics of IV tocilizumab plus GC in patients with active MPA and GPA compared to treatment with IVCY plus GC. The trial is designed to show the non-inferiority of the former. The primary endpoint is the percentage of patients achieving BVAS remission (BVAS $=0$ at two consecutive visits made at least 1 month apart) and a PSL dose of $7.5 \mathrm{mg} /$ day at week 24 (BVAS and GC remission). The key secondary endpoints are: the percentages of patients who maintain BVAS and GC remission from week 24 to week 52 and the time to failure to maintain BVAS and GC remission from week 24; the percentages of patients achieving BVAS remission and the time to BVAS remission by week 24; the percentages of patients able to taper PSL to $7.5 \mathrm{mg} /$ day and the time to that dosage by week 24; and the accumulated doses of PSL by weeks 24 and 52. Drug safety and pharmacokinetics parameters will also be evaluated.

The eligibility criteria are summarized in Table 3. A patient must fulfill the Ministry of Health, Labour, and Welfare diagnostic criteria of MPA and GPA [44] and be positive for ANCA or have renal-limited vasculitis with pauci-immune type glomerulonephritis confirmed by renal biopsy and ANCA positivity. After dynamic allocation with adjustment for ANCA subtypes and serum levels of creatinine, patients randomized to the tocilizumab plus GC group will receive IV 8 mg tocilizumab/ $\mathrm{kg}$ biweekly from week o to week 16 and every 4 weeks from week 20 to week 48. Patients who do not achieve remission by week 16 will continue biweekly tocilizumab up to week 24 and change every 4 weeks onward. Patients randomized to the IVCY plus GC group will receive three to six IV infusions of CY at $15 \mathrm{mg} / \mathrm{kg}$ at intervals of 2 to 4 weeks according to the discretion of the investigators. Four weeks after the last infusion, if a patient has achieved BVAS $=0$, IVCY will be replaced by $1.5 \mathrm{mg}$ azathioprine/kg per day until week 52 . All en- 


\section{KJIM ${ }^{-}$}

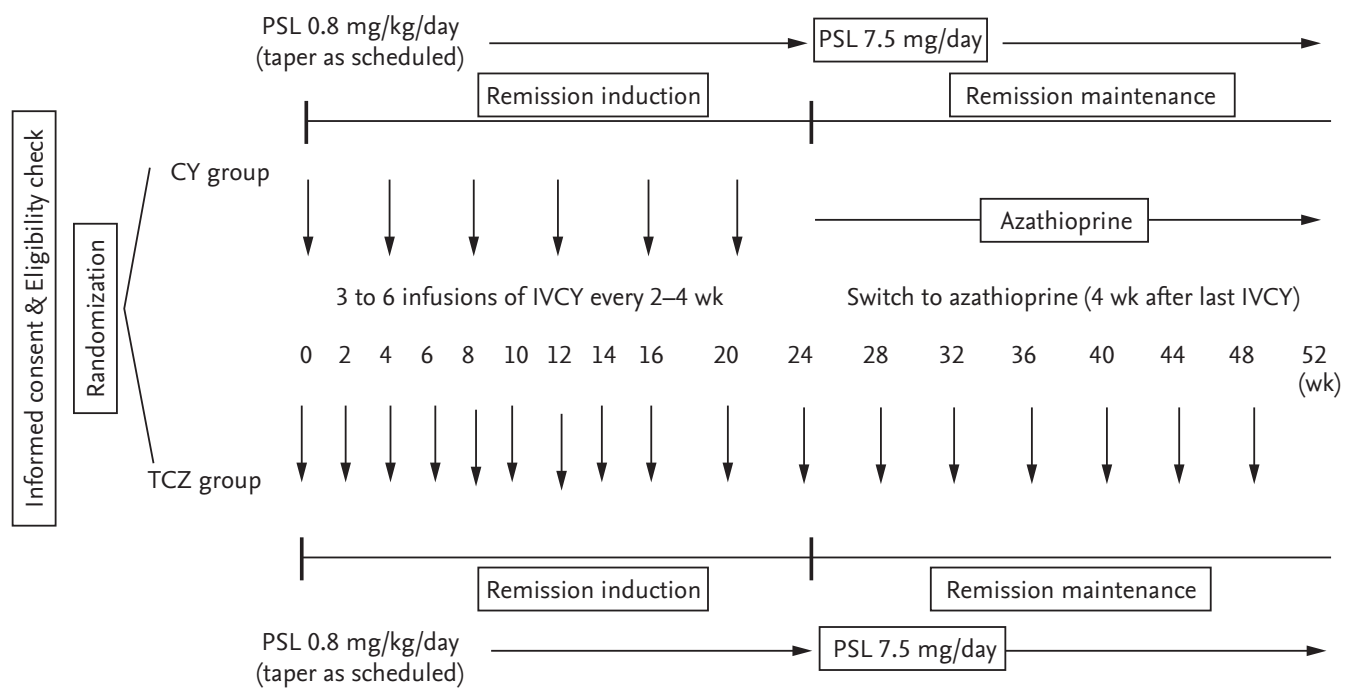

Figure 1. Flow chart of the randomized controlled trial of tocilizumab (TCZ) for the treatment of microscopic polyangiitis (MPA) and granulomatosis with polyangiitis (GPA). Patients meeting the eligible criteria (Table 3) are randomized into two groups, receiving intravenous TCZ plus prednisolone (PSL) or intravenous cyclophosphamide (CY) plus PSL, and are followed up to week 52. The specific dosages and treatment intervals are described in the text. The initial PSL dose is $0.8 \mathrm{mg} / \mathrm{kg} / \mathrm{day}$, tapered to 7.5 $\mathrm{mg} /$ day by week 24 according to the predefined schedule and then maintained up to week 52 . The primary endpoint is the percentage of patients achieving Birmingham Vasculitis Activity Score (BVAS) remission (BVAS $=0$ at two consecutive visits made at least 1 month apart) and PSL efficacy at $7.5 \mathrm{mg}$ /day at week 24. IVCY, intravenous cyclophosphamide.

Table 3. Eligibility criteria for the randomized controlled trial of tocilizumab for MPA and GPA

1. Meeting the diagnostic criteria of MPA and GPA by the Ministry of Health, Labour, and Welfare of Japan and being positive for ANCA, or having renal limited vasculitis with pauci-immune type glomerulonephritis by renal biopsy and ANCA positivity

2. $\geq 20$ years old and $<85$ years old

3. Body weight $\geq 40 \mathrm{~kg}$

4. Birmingham Vasculitis Activity Score $\geq 3$ with predefined organ involvement or necessitating treatment with CY

5. C-reactive protein $\geq 1.0 \mathrm{mg} / \mathrm{dL}$

6. Agreeing to the use of contraception during the clinical trial and for a predefined period after the clinical trial

7. Agreeing to not breast feed a baby during the clinical trial

8. Being able to follow the clinical trial protocol

9. Being able to obtain written informed consent

MPA, microscopic polyangiitis; GPA, granulomatosis with polyangiitis; ANCA, anti-neutrophil cytoplasmic antibody; CY, cyclophosphamide.

${ }^{a}$ Eligible patients meet all of the above criteria.

rolled patients will receive an initial PSL dosage of 0.8 $\mathrm{mg} / \mathrm{kg} /$ day, which will be tapered as scheduled to 7.5 mg/day by week 24 and continued until week 52. For patients who do not achieve the primary endpoint by week 24, treatment with the test drug will be discontin- ued and treatment will proceed based on the best medical judgment of investigators.

Our estimate of the BVAS and GC remission rate at week 24 is $90 \%$ in the tocilizumab plus GC group and $80 \%$ in the IVCY plus GC group. The non-inferiority 
margin was set at $20 \%$ with one-sided alpha $=0.025$ and beta $=0.2$. Assuming a dropout rate of 10\%, 24 patients for each arm or 48 patients in total must be enrolled to demonstrate the non-inferiority of tocilizumab plus GC compared to IVCY plus GC. The trial is scheduled to be completed by March 2022.

\section{CONCLUSIONS}

Although the results of ongoing or scheduled clinical trials are currently pending, the clinical development of molecular targeted therapy for MPA and GPA is promising and is expected to rapidly transform treatment so that it will be GC-free or at least less GC-dependent. Decreasing or eliminating the use of GC will reduce the burden of accrued damage and improve the longterm outcomes for patients. However, several pertinent clinical questions about molecular targeted therapy for MPA and GPA still need to be addressed: Are there subgroups of patients who respond better to one molecular targeted therapy than to others? Which molecular targeted therapy should be used first? Are good biomarkers available for each form of molecular targeted therapy? Can treatment with molecular targeted therapy be discontinued or switched to conventional immunosuppressants without increasing the risk for major flares? Can patients be treated with molecular targeted therapies at a reasonable cost? Analyses of multi-national, national, or regional registries, claims databases, and the results of well-designed clinical trials should provide answers to these questions.

\section{Conflict of interest}

No potential conflict of interest relevant to this article was reported.

\section{REFERENCES}

1. Hoffman GS, Kerr GS, Leavitt RY, et al. Wegener granulomatosis: an analysis of 158 patients. Ann Intern Med 1992;116:488-498.

2. Nachman PH, Hogan SL, Jennette JC, Falk RJ. Treatment response and relapse in antineutrophil cytoplasmic autoantibody-associated microscopic polyangiitis and glo- merulonephritis. J Am Soc Nephrol 1996;7:33-39.

3. Harigai M, Nagasaka K, Amano K, et al. 2017 Clinical practice guidelines of the Japan Research Committee of the Ministry of Health, Labour, and Welfare for Intractable Vasculitis for the management of ANCA-associated vasculitis. Mod Rheumatol 2019;29:20-30.

4. Ntatsaki E, Carruthers D, Chakravarty K, et al. BSR and BHPR guideline for the management of adults with ANCA-associated vasculitis. Rheumatology (Oxford) 2014;53:2306-2309.

5. Yates M, Watts RA, Bajema IM, et al. EULAR/ERA-EDTA recommendations for the management of ANCA-associated vasculitis. Ann Rheum Dis 2016;75:1583-1594.

6. Kidney Disease Improving Global Outcomes. KDIGO Clinical Practice Guidelines for Glomerulonephritis. Kidney Int Suppl 2012;2:139-274.

7. Smolen JS, Aletaha D, McInnes IB. Rheumatoid arthritis. Lancet 2016;388:2023-2038.

8. Nagasaka K, Harigai M, Hagino N, et al. Systematic review and meta-analysis for 2017 clinical practice guidelines of the Japan Research Committee of the Ministry of Health, Labour, and Welfare for Intractable Vasculitis for the management of ANCA-associated vasculitis. Mod Rheumatol 2019;29:119-129.

9. Takahira S. Comparison of treatment with corticosteroid alone vs corticosteroid and cyclophosphamide in combination in patients with MPO-ANCA positive necrotizing crescentic glomerulonephritis during 5 years follow-up. J Saitama Med Univ 2002;29:221-228.

10. Nakaya I, Yahata M, Takahashi S, et al. Long-term outcome and efficacy of cyclophosphamide therapy in Japanese patients with ANCA-associated microscopic polyangiitis: a retrospective study. Intern Med 2013;52:25032509.

11. de Groot K, Harper L, Jayne DR, et al. Pulse versus daily oral cyclophosphamide for induction of remission in antineutrophil cytoplasmic antibody-associated vasculitis: a randomized trial. Ann Intern Med 2009;150:670-680.

12. Haubitz M, Schellong S, Gobel U, et al. Intravenous pulse administration of cyclophosphamide versus daily oral treatment in patients with antineutrophil cytoplasmic antibody-associated vasculitis and renal involvement: a prospective, randomized study. Arthritis Rheum 1998;41:1835-1844.

13. Guillevin L, Cordier JF, Lhote F, et al. A prospective, multicenter, randomized trial comparing steroids and pulse 
cyclophosphamide versus steroids and oral cyclophosphamide in the treatment of generalized Wegener's granulomatosis. Arthritis Rheum 1997;40:2187-2198.

14. Jones RB, Tervaert JW, Hauser T, et al. Rituximab versus cyclophosphamide in ANCA-associated renal vasculitis. $\mathrm{N}$ Engl J Med 2010;363:211-220.

15. Stone JH, Merkel PA, Spiera R, et al. Rituximab versus cyclophosphamide for ANCA-associated vasculitis. N Engl J Med 2010;363:221-232.

16. Robson J, Doll H, Suppiah R, et al. Damage in the ANCA-associated vasculitides: long-term data from the European Vasculitis Study Group (EUVAS) therapeutic trials. Ann Rheum Dis 2015;74:177-184.

17. Specks U, Merkel PA, Seo P, et al. Efficacy of remission-induction regimens for ANCA-associated vasculitis. N Engl J Med 2013;369:417-427.

18. Jones RB, Furuta S, Tervaert JW, et al. Rituximab versus cyclophosphamide in ANCA-associated renal vasculitis: 2-year results of a randomised trial. Ann Rheum Dis 2015;74:1178-1182.

19. Guillevin L, Pagnoux C, Karras A, et al. Rituximab versus azathioprine for maintenance in ANCA-associated vasculitis. N Engl J Med 2014;371:1771-1780.

20. Terrier B, Pagnoux C, Perrodeau E, et al. Long-term efficacy of remission-maintenance regimens for ANCA-associated vasculitides. Ann Rheum Dis 2018;77:1150-1156.

21. Charles P, Terrier B, Perrodeau E, et al. Comparison of individually tailored versus fixed-schedule rituximab regimen to maintain ANCA-associated vasculitis remission: results of a multicentre, randomized controlled, phase III trial (MAINRITSAN2). Ann Rheum Dis 2018;77:1143-1149.

22. Schneeweis C, Rafalowicz M, Feist E, et al. Increased levels of BLyS and sVCAM-1 in anti-neutrophil cytoplasmatic antibody (ANCA)-associated vasculitides (AAV). Clin Exp Rheumatol 2010;28(1 Suppl 57):62-66.

23. Nagai M, Hirayama K, Ebihara I, Shimohata H, Kobayashi M, Koyama A. Serum levels of BAFF and APRIL in myeloperoxidase anti-neutrophil cytoplasmic autoantibody-associated renal vasculitis: association with disease activity. Nephron Clin Pract 2011;118:c339-c345.

24. Xin G, Chen M, Su Y, Xu LX, Zhao MH, Li KS. Serum B-cell activating factor in myeloperoxiase-antineutrophil cytoplasmic antibodies-associated vasculitis. Am J Med Sci 2014;348:25-29.

25. McClure M, Gopaluni S, Jayne D, Jones R. B cell therapy in ANCA-associated vasculitis: current and emerging treatment options. Nat Rev Rheumatol 2018;14:580-591.

26. Gerard C, Gerard NP. C5A anaphylatoxin and its seven transmembrane-segment receptor. Annu Rev Immunol 1994;12:775-808.

27. Chen M, Jayne DRW, Zhao MH. Complement in ANCA-associated vasculitis: mechanisms and implications for management. Nat Rev Nephrol 2017;13:359-367.

28. Bekker P, Dairaghi D, Seitz L, et al. Characterization of pharmacologic and pharmacokinetic properties of ccxi68, a potent and selective orally administered complement 5a receptor inhibitor, based on preclinical evaluation and randomized phase 1 clinical study. PLoS One 2016;11:e0164646.

29. Jayne DRW, Bruchfeld AN, Harper L, et al. Randomized trial of $\mathrm{C}_{5}$ a receptor inhibitor avacopan in ANCA-associated vasculitis. J Am Soc Nephrol 2017;28:2756-2767.

30. Xiao H, Dairaghi DJ, Powers JP, et al. C5a receptor (CD88) blockade protects against MPO-ANCA GN. J Am Soc Nephrol 2014;25:225-231.

31. ClinicalTrials.gov. A phase 3 clinical trial of CCXı68 (Avacopan) in patients with ANCA-associated vasculitis (ADVOCATE) [Internet]. Bethesda (MD): U.S. National Library of Medicine, 2018 [cited 2018 Nov 15]. Available from: https://clinicaltrials.gov/ct2/show/NCTo2994927?term=CCX168\&cond=ANCA+Associated+Vasculitis\&rank=1.

32. Nagao T, Kusunoki R, Iwamura C, et al. Correlation of interleukin-6 and monocyte chemotactic protein-1 concentrations with crescent formation and myeloperoxidase-specific anti-neutrophil cytoplasmic antibody titer in SCG/Kj mice by treatment with anti-interleukin-6 receptor antibody or mizoribine. Microbiol Immunol 2013:57:640-650.

33. Kekow J, Szymkowiak CH, Sticherling M, Schroder JM, Christophers E, Gross WL. Pro- and anti-inflammatory cytokines in primary systemic vasculitis. Adv Exp Med Biol 1993;336:341-344.

34. Muller Kobold AC, van Wijk RT, Franssen CF, et al. In vitro up-regulation of E-selectin and induction of interleukin-6 in endothelial cells by autoantibodies in Wegener's granulomatosis and microscopic polyangiitis. Clin Exp Rheumatol 1999;17:433-440.

35. Popa ER, Franssen CF, Limburg PC, Huitema MG, Kallenberg CG, Tervaert JW. In vitro cytokine production and proliferation of $\mathrm{T}$ cells from patients with anti-proteinase 3- and antimyeloperoxidase-associated vasculitis, in response to proteinase 3 and myeloperoxidase. Arthri- 
tis Rheum 2002;46:1894-1904.

36. Ohlsson S, Wieslander J, Segelmark M. Circulating cytokine profile in anti-neutrophilic cytoplasmatic autoantibody-associated vasculitis: prediction of outcome? Mediators Inflamm 2004;13:275-283.

37. Tsutsumino M, Sada K, Arimua Y, Makino H, Harigai M, Yamanaka H. Interluekin- 6 as a therapeutic target in patients with anti-neutrophil cytoplasmic antibody-associated vasculitis. Mod Rheumatol 2017;27(Suppl 2017):S245.

38. Berti A, Cavalli G, Campochiaro C, et al. Interleukin-6 in ANCA-associated vasculitis: rationale for successful treatment with tocilizumab. Semin Arthritis Rheum 2015;45:48-54.

39. Takenaka K, Ohba T, Suhara K, Sato Y, Nagasaka K. Successful treatment of refractory aortitis in antineutrophil cytoplasmic antibody-associated vasculitis using tocilizumab. Clin Rheumatol 2014;33:287-289.

40. Sakai R, Kondo T, Kurasawa T, et al. Current clinical evidence of tocilizumab for the treatment of ANCA-associated vasculitis: a prospective case series for microscopic polyangiitis in a combination with corticosteroids and literature review. Clin Rheumatol 2017;36:2383-2392.

41. Sumida K, Ubara Y, Suwabe T, et al. Complete remission of myeloperoxidase-anti-neutrophil cytoplasmic antibody-associated crescentic glomerulonephritis complicated with rheumatoid arthritis using a humanized anti-interleukin 6 receptor antibody. Rheumatology (Oxford) 2011;50:1928-1930.

42. Sakai R, Shibata A, Chino K, et al. Corticosteroid- and cyclophosphamide-free treatment of anti-neutrophil cytoplasmic antibody-associated vasculitis using tocilizumab. Mod Rheumatol 2015;25:810-811.

43. Watts R, Lane S, Hanslik T, et al. Development and validation of a consensus methodology for the classification of the ANCA-associated vasculitides and polyarteritis nodosa for epidemiological studies. Ann Rheum Dis 2007;66:222-227.

44. Sada KE, Yamamura M, Harigai M, et al. Issues associated with the Ministry of Health, Labour and Welfare diagnostic criteria for antineutrophil cytoplasmic antibody-associated vasculitides: reclassification of patients in the prospective cohort study of Remission Induction Therapy in Japanese patients with ANCA-associated vasculitides according to the MHLW criteria. Mod Rheumatol 2015;25:657-659. 